\title{
miR-196b-5p Promotes Proliferation, Migration and Invasion of Lung Adenocarcinoma Cells via Targeting RSPO2
}

This article was published in the following Dove Press journal: Cancer Management and Research

\author{
Qian $X u^{\prime}$ \\ Zhenwu $\mathrm{Xu}^{2}$ \\ 'Department of Oncology Medicine, \\ Fujian Medical University Union Hospital, \\ Fuzhou, 35000I, People's Republic of \\ China; ${ }^{2}$ Department of Thoracic Medical \\ Oncology, Fujian Provincial Cancer \\ Hospital, The Affiliated Hospital of Fujian \\ Medical University, Fuzhou, 3500I4, \\ People's Republic of China
}

Objective: To explore the biological role of miR-196b-5p/RSPO2 in the occurrence and development of lung adenocarcinoma (LUAD) and to provide a basis for finding new therapeutic targets for LUAD.

Methods: Differentially expressed genes were analyzed based on LUAD microarray, and the target gene of the target miRNA was predicted. qRT-PCR was used to detect the expression levels of miR-196b-5p and RSPO2 mRNA in normal human bronchial epithelial cell line BEAS-2B and LUAD cell lines A549, NCI-H1792 and NCI-H226. Western blot was used to evaluate protein expression. Cell proliferative, migratory and invasive abilities were detected by CCK- 8 and transwell assays. Dual-luciferase assay was conducted to verify the targeting relationship between miR-196b-5p and RSPO2.

Results: The results of qRT-PCR showed that miR-196b-5p was significantly highly expressed in LUAD cells, and the expression level of its downstream target gene RSPO2 was significantly decreased. The results of CCK-8 and transwell assays exhibited that miR-196b-5p promoted proliferation, migration and invasion of LUAD cells, while RSPO2 inhibited the malignant progression of LUAD cells. Dual-luciferase assay confirmed the targeted binding relationship between miR-196b-5p and RSPO2. Overexpression of RSPO2 partially reversed the promotion of miR-196b-5p on proliferation, migration and invasion of LUAD cells.

Conclusion: miR-196b-5p promoted proliferation, migration and invasion of LUAD cells by targeting and down-regulating RSPO2, which provided ideas for searching new targets for the diagnosis and treatment of LUAD.

Keywords: RSPO2, miR-196b-5p, lung adenocarcinoma, proliferation, invasion

\section{Introduction}

At present, lung cancer is the most common malignant tumor in the world with an extremely high rate of morbidity and mortality, which poses a great threat to human health. ${ }^{1-3}$ Non-small cell lung cancer (NSCLC) accounts for about $85 \%$ of all lung cancer cases, and lung adenocarcinoma (LUAD) is the most common histological subtype of NSCLC, accounting for $40 \%$ to $50 \%$ of the diagnosed NSCLC. ${ }^{4,5}$ In recent years, molecular targeted therapy for lung cancer has developed rapidly, and the in-depth study of miRNAs and related regulatory axes provide a new direction for the diagnosis and treatment of lung cancer. However, due to postoperative metastatic recurrence and other factors, the 5-year survival rate of LUAD patients is still not optimistic., Therefore, it is of great practical significance to further look for new diagnostic markers and therapeutic targets for LUAD.
Correspondence: Zhenwu Xu

Oncology, Fujian Provincial Cancer

Hospital, The Affiliated Hospital of Fujian

Medical University, No. 420 Fuma Road,

Fuzhou 350014, People's Republic of

China

Tel +86-13906931946

Email xuzhenwu32540@163.com

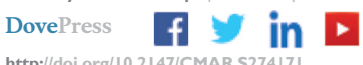

http://doi.org//0.2147/CMAR.S274174
Cancer Management and Research 2020:12 13393-13402

13393

(c) (i) (\$) $\odot 2020 \mathrm{Xu}$ and $\mathrm{Xu}$. This work is published and licensed by Dove Medical Press Limited. The full terms of this license are available at https://www.dovepress.com/terms.php

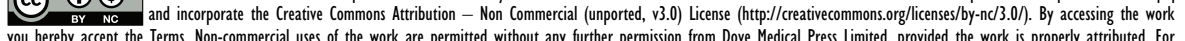
you hereby accept the Terms. Non-commercial uses of the work are permitted without any further permision from Dove Medical Press Limited, provided the work is properly attributed. For permission for commercial use of this work, please see paragraphs 4.2 and 5 of our Terms (https://www.dovepress.com/terms.php). 
MicroRNAs (miRNAs) are endogenous non-coding small RNAs involved in post-transcriptional regulation of genes, and they can regulate a variety of biological functions of cells. ${ }^{8,9}$ The aberrant expression of miRNAs is often observed in tumor cells, and different miRNAs may play a role of an oncogene or a tumor suppressor gene in the same cancer type. miR-200b-3p as an oncogene is upregulated in LUAD cells and promotes cell proliferation and migration. ${ }^{10}$ miR-1290 is also highly expressed in LUAD cells and promotes LUAD cell proliferation and invasion by targeting and down-regulating SOCS4. ${ }^{11} \mathrm{miR}$ 1827 is down-regulated in LUAD and functions as a tumor suppressor to inhibit the development of LUAD.${ }^{12}$ However, the mechanism of miR-196b-5p in LUAD is still unclear.

R-Spondin (RSPO) family of secreted proteins plays a critical role in embryonic development and organogenesis. ${ }^{13}$ It has been reported that the RSPO family can regulate cell processes and has been proved to be related to the occurrence and development of tumors. ${ }^{14,15} \mathrm{RSPO} 2$ plays a key role in the development of limbs, laryngotracheal and lungs. ${ }^{16}$ Recent studies have reported the dual effect of RSPO2 abnormal expression on tumor development. Yin et al found that RSPO2 is highly expressed in hepatocellular carcinoma tissue and cells, and Zhang et al also discovered that RSPO2 is up-regulated in gastric cancer, and overexpression of RSPO2 can promote proliferation and metastasis of hepatocellular carcinoma and gastric cancer cells. ${ }^{17,18}$ Meanwhile, the antitumor effect of RSPO2 should not be ignored. Wu et al reported that RSPO2 functions as a tumor suppressor in human colorectal cancer, and overexpression of RSPO2 can inhibit proliferation and tumorigenicity of colorectal cancer cells, whereas down-regulation of RSPO2 can enhance tumor cell growth. ${ }^{19}$ In addition, a study first reported that the upregulation of RSPO2 indicates a good prognosis of LUAD patients. $^{13}$

This study firstly explored the expression patterns and biological functions of miR-196b-5p and RSPO2 in LUAD, and designed experiments to prove the role of the miR-196b-5p/RSPO2 regulatory axis in the development of LUAD, which is of profound significance to find new targets for the diagnosis and treatment of LUAD.

\section{Materials and Methods Bioinformatics Analysis}

The expression profiles of mature miRNAs (including 46 normal samples and 521 tumor samples) and mRNAs (including 59 normal samples and 535 tumor samples) of LUAD were downloaded from The Cancer Genome Atlas (TCGA) database (https://portal.gdc.cancer.gov/). Differentially expressed genes (DEGs) were obtained by differential analysis using EdgeR with $|\operatorname{logFC}|>2.0$ and padj $<0.01$ as the threshold based on the downloaded data. miRDB (http://mirdb.org/), mirDIP (http://ophid.utoronto.ca/mirDIP/index.jsp\#r), TargetScan (http://www.targetscan.org/vert 72/) and starBase (http://starbase.sysu.edu.cn/) databases were used to predict target genes of the target miRNA. After taking intersection of the predicted target genes and down-regulated differentially expressed mRNAs (DEmRNAs), the mRNA with the highest correlation was selected for study. One-way analysis of variance (ANOVA) was used to analyze the correlation between target mRNA expression and clinical stage of patients with LUAD (stage I: $n=268$; stage II: $n=120$; stage III: $n=81$; stage IV: $n=26$ ). The clinical information of patients in the TCGALUAD dataset is detailed in Supplementary Material 1.

\section{Cell Culture}

Human normal bronchial epithelial cell line BEAS-2B (BNCC338205) and LUAD cell lines A549 (BNCC337696), NCI-H1792 (BNCC338328) and NCI-H226 (BNCC100405) were purchased from BeNa Culture Collection (Beijing, China). BEAS-2B and A549 cells were cultured in high glucose Dulbecco's Modified Eagle's Medium (DMEM; Sigma, St. Louis, MO, USA) with $10 \%$ fetal bovine serum (FBS; Sigma, St. Louis, MO, USA). NCI-H1792 and NCI-H226 cells were cultured in RPMI-1640 (Sigma, St. Louis, MO, USA) with $10 \% \mathrm{FBS}$. All cells were cultured at $37^{\circ} \mathrm{C}$ with $5 \%$ $\mathrm{CO}_{2}$.

\section{Quantitative Real-Time PCR (qRT-PCR)}

Total RNA was extracted from various cell lines by TRIzol reagent (Life Technologies corporation, Gaithersburg, MD, USA). Reverse transcription was performed using PrimeScript II 1st Strand cDNA Synthesis Kit (TaKaRa, Tokyo, Japan) according to the instructions, and qRT-PCR was conducted using SYBR Premix Ex Taq (TaKaRa). U6 and GAPDH were used as internal regulators of miR-196b-5p and RSPO2, respectively, to analyze the relative expression. The relative expression levels of miR-196b-5p and RSPO2 mRNA were calculated by $2^{-\Delta \Delta \mathrm{Ct}}$ method. The primer sequences used in the experiments are listed in Table 1.

\section{Cell Transfection}

miR-196b-5p mimic, miR-196b-5p inhibitor, NC mimic, NC inhibitor, NC siRNA and RSPO2 siRNA were purchased from RiboBio (RiboBio, Guangzhou, Guangdong, China). 
Table I Primer Sequences for qRT-PCR

\begin{tabular}{|l|l|}
\hline Gene & Primer Sequence \\
\hline miR-196b-5p & $\begin{array}{l}\text { F: 5' -GCGCGTAGGTAGTTTCCTGTT-3' } \\
\text { R: 5'-AGTGCAGGGTCCGAGGTATT-3' }\end{array}$ \\
\hline RSPO2 & $\begin{array}{l}\text { F: 5'-GGGTCACTGAAGAAGCTACTAC-3' } \\
\text { R: 5'-ACTGCTCTGAAAGTCACCTG-3' }\end{array}$ \\
\hline GAPDH & F: 5'-TGGTCACCAGGGCTGCTT-3' \\
& R: 5'-AGCTTCCCGTTCTCAGCC-3' \\
\hline U6 & $\begin{array}{l}\text { R: 5'-CTCGCTTCGGCAGCACA-3' } \\
\text { R: 5'-AACGCTTCACGAATTTGCGT-3' }\end{array}$ \\
\hline
\end{tabular}

LUAD cells were incubated in a 6-well plate for $24 \mathrm{~h}$ before transfection and the transfection was conducted using Lipofectamine 2000 transfection reagent (Invitrogen). After $48 \mathrm{~h}$, the transfection efficiency was examined and the cells were used for subsequent experiments.

\section{CCK-8 Assay}

The transfected cells were inoculated into a 96-well plate with $1 \times 10^{3}$ cells/well. At $24 \mathrm{~h}, 48 \mathrm{~h}, 72 \mathrm{~h}$ and $96 \mathrm{~h}$, mixture of $10 \mu \mathrm{L}$ CCK- 8 solution and $100 \mu \mathrm{L}$ medium was added to the plate. After incubation at $37^{\circ} \mathrm{C}$ for $2 \mathrm{~h}$, the absorbance value at $450 \mathrm{~nm}$ was detected by a multifunctional enzyme-linked analyzer FLx8 (BioTek, Vermont, USA). The absorbance at each time point was measured and the curve graph of the absorbance was plotted.

\section{Transwell Assay}

Cell migratory and invasive abilities were determined using transwell chambers with polycarbonate membrane ( 8 - $\mu \mathrm{m}$ pore size). In the migration experiment, $1 \times 10^{5}$ cells were inoculated into the upper chamber without Matrigel. In the invasion experiment, Matrigel (BD Bioscience, San Jose, CA, USA) diluted by serum-free medium was added to the upper chamber. After the Matrigel was solidified at $37^{\circ} \mathrm{C}, 200 \mu \mathrm{L}$ of serum-free medium was added for Matrigel hydration and $1 \times 10^{5}$ cells were inoculated into the upper chamber. In the lower chamber, complete medium containing $20 \%$ FBS was added as attractant. After culture for $24 \mathrm{~h}$ at $37^{\circ} \mathrm{C}$ with $5 \% \mathrm{CO}_{2}$, the Matrigel and cells in the upper chamber and the medium were removed, while the cells in the lower chamber were fixed with methanol, and then stained with $0.1 \%$ crystal violet dye for $30 \mathrm{~min}$. Finally, the migratory and invading cells were observed and the numbers were counted under the microscope (Olympus, Tokyo, Japan), respectively.

\section{Dual-Luciferase Assay}

TargetScan database was used to predict the targeted binding sites of miR-196b-5p on RSPO2 3'-untranslated regions (3'UTR). In order to further verify the targeted binding relationship between miR-196b-5p and RSPO2, we conducted the dual-luciferase assay. The wild-type (WT) and mutant-type (MUT) RSPO2 3'-UTR were cloned into the pGL3 promoter vector to construct WT-RSPO2 and MUT-RSPO2. The constructed vectors and miR-196b-5p mimic or NC mimic were co-transfected into LUAD cells using Lipofectamine 2000. The luciferase activity was determined by Dual-Luciferase Reporter Assay Kit (Beyotime Biotechnology, Shanghai, China) after $48 \mathrm{~h}$ of culture.

\section{Western Blot}

Total proteins were extracted from various cell lines using RIPA lysis buffer (KEYGENE, Nanjing, China), separated by $10 \%$ SDS-PAGE, and transferred onto the PVDF membrane. The membrane was blocked in TBST containing 5\% skim milk powder for $1 \mathrm{~h}$ at room temperature, and then was incubated with rabbit polyclonal RSPO2 antibody (Cat. No. ab73761; dilution, 1:1000; Abcam) and rabbit polyclonal $\beta$ actin antibody (Cat. No.10303001; dilution, 1:5000; Harmonious One Biotechnology, Shanghai, China) at $4^{\circ} \mathrm{C}$ overnight. Horseradish peroxidase-conjugated secondary antibody anti-rabbit IgG (Cat. No. 7074; dilution, 1:3000; Cell Signaling Technology, Inc.) was added to the membrane for 1 h of culture. Finally, FluorChem M System (Protein Simple, San Jose, CA) was used to detect protein expression levels.

\section{Statistical Analysis}

All data were processed by GraphPad 8.0 statistical software, and the measurement data were expressed as mean \pm standard deviation. The comparison between two groups was analyzed by $t$-test. NS represented no statistical significance, $p<0.05$ indicated statistical significance, $p<0.01$ indicated highly statistical significance and $p<0.001$ indicated extremely statistical significance.

\section{Results miR-196b-5p is Highly Expressed in LUAD Tissue and Cells}

A total of 2495 DEGs were obtained by differential analysis using EdgeR, among which 1968 were up-regulated and 527 were down-regulated (Figure 1A). Data of TCGA-LUAD showed that miR-196b-5p was significantly up-regulated in LUAD tissue (Figure 1B). qRT-PCR was 
used to further detect the expression level of miR-196b-5p in normal bronchial epithelial cell line and LUAD cell lines. The results showed that compared with BEAS-2B, miR-196b-5p was significantly up-regulated in LUAD cell lines, and the highest expression of miR-196b-5p was observed in A549 cell line (Figure 1C)

\section{miR-196b-5p Can Promote Proliferation, Migration and Invasion of LUAD Cells}

miR-196b-5p was significantly up-regulated in LUAD tissue and cells. To explore the biological effect of miR196b-5p on LUAD cells, A549 cell line was selected for subsequent experiments. Firstly, the transfection efficiency was detected by measuring the expression of miR-196b-5p in the transfection groups of NC mimic, $\mathrm{NC}$ inhibitor, miR-196b-5p mimic and miR-196b-5p inhibitor. The results exhibited that miR-196b-5p was significantly upregulated in cancer cells transfected with miR-196b-5p mimic, and it was greatly down-regulated in cancer cells transfected with miR-196b-5p inhibitor, compared with their respective controls, indicating a successful transfection (Figure 2A).

The proliferation of A549 cells was detected by CCK- 8 assay. The results suggested that the absorbance of the miR-196b-5p mimic group was higher than that of the NC mimic group under the same culture conditions (Figure 2B), and the absorbance of the miR-196b$5 p$ inhibitor group was lower than that of the NC inhibitor group (Figure 2C). It indicated that overexpression of miR-196b-5p promoted cell proliferative capacity, while knock down of miR-196b-5p inhibited cell proliferation. Transwell assay (Figure 2D and E) revealed that overexpression of miR-196b-5p significantly enhanced cell migration and invasion, while inhibiting miR-196b-5p caused opposite results. The above results indicated that overexpression of miR-196b-5p could promote proliferation, migration and invasion of LUAD cells, while down-regulation of miR-196b-5p played an inhibitory role in LUAD cells.

\section{miR-196b-5p Targets and Down-Regulates RSPO2}

Target genes of miR-196b-5p were predicted by TargetScan, miRDB, mirDIP and starBase databases, and three candidate target genes were obtained by intersection with downregulated DEmRNAs (Figure 3A). Pearson correlation analysis of miR-196b-5p and the three target genes were conducted and the most significant correlation was observed between miR-196b-5p and RSPO2 (Figure 3B and C). Moreover, bioinformatics analysis exhibited that RSPO2 was significantly down-regulated in LUAD (Figure 3D). It was further revealed that the expression of RSPO2 was marginally associated with clinical stage of patients with LUAD (Figure 3E). To verify the results of bioinformatics, qRT-PCR was used to determine the expression of RSPO2 in BEAS-2B cell line and LUAD cell lines. The mRNA expression of RSPO2 in 3 LUAD cell lines was found to be significantly down-regulated compared with that in BEAS2B cells (Figure 3F).

Based on the prediction, RSPO2 was the target gene of miR-196b-5p, and the targeted binding sites are shown in Figure 3G. Dual-luciferase reporter assay was performed to confirm the targeted relationship. The results revealed that overexpression of miR-196b-5p significantly reduced the luciferase activity of WT-RSPO2, but no inhibition effect was found in the MUT-RSPO2 group, suggesting
A

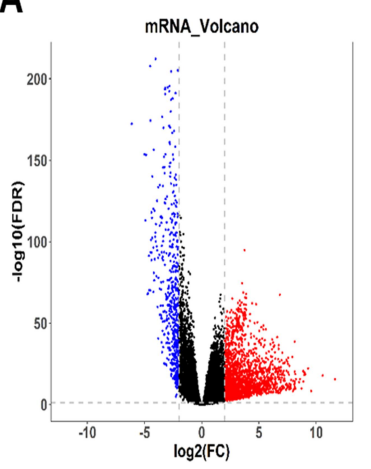

B

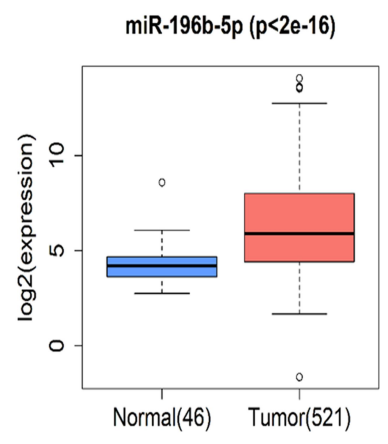

C

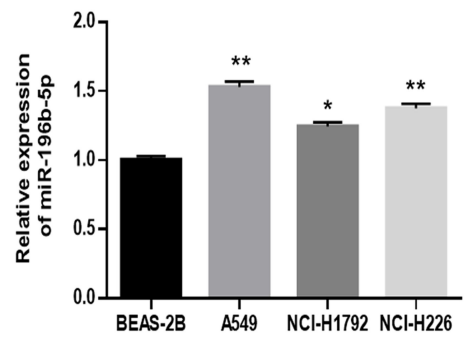

Figure I miR-196b-5p is highly expressed in LUAD tissue and cells. (A) Volcano map of DEmRNAs in TCGA-LUAD. (B) The box plot of miR-I96b-5p expression in normal tissue (blue) and tumor tissue (red). (C) The expression of miR-196b-5p in human normal bronchial epithelial cell line and three LUAD cell lines was detected by qRT-PCR.. All results were representative of three independent experiments. $* p<0.05, * * p<0.0$ I. 

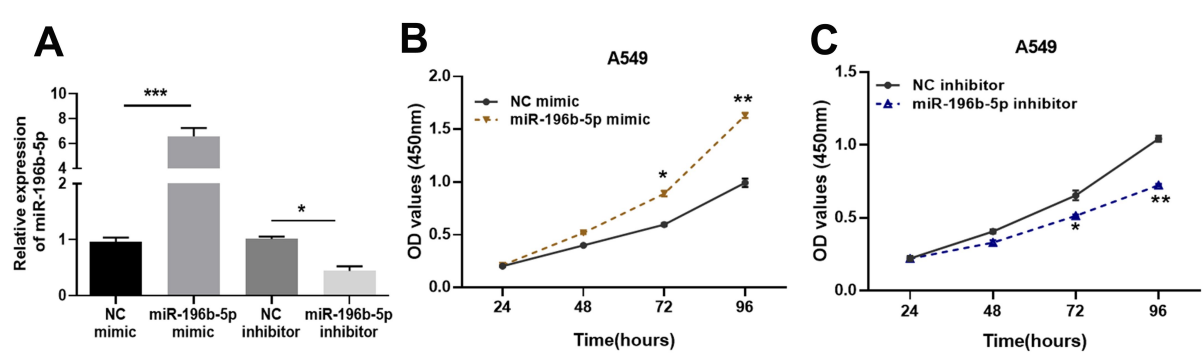

D
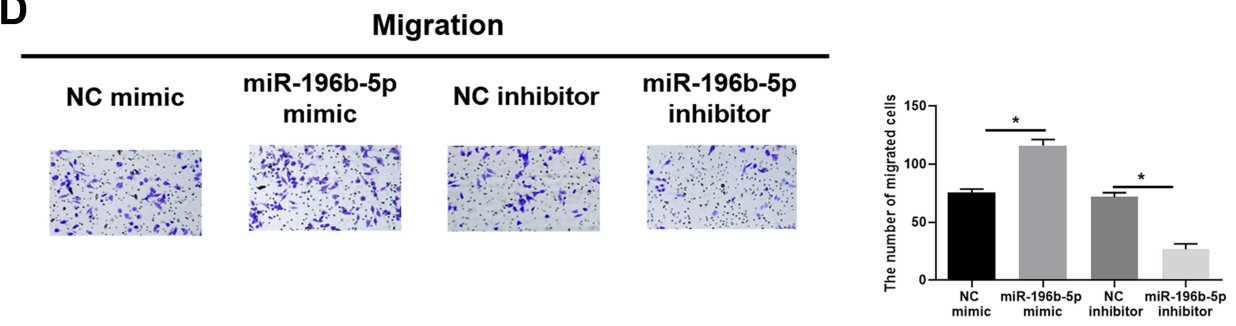

E

Invasion

\begin{tabular}{|c|c|c|c|}
\hline NC mimic & $\begin{array}{c}\text { miR-196b-5p } \\
\text { mimic }\end{array}$ & NC inhibitor & $\begin{array}{l}\text { miR-196b-5p } \\
\text { inhibitor }\end{array}$ \\
\hline$x^{3}$ & 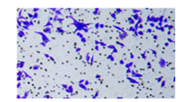 & 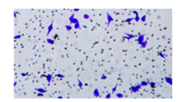 & \\
\hline
\end{tabular}

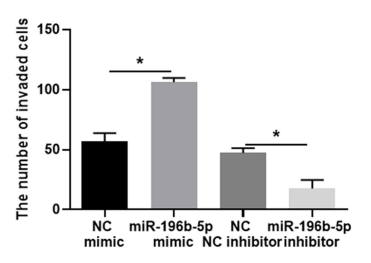

Figure 2 miR-196b-5p can promote proliferation, migration and invasion of LUAD cells. (A) miR-196b-5p expression in A549 cells transfected with miR-196b-5p mimic or miR-196b-5p inhibitor. (B and C) Cell viability of cells in the miR-196b-5p mimic and miR-196b-5p inhibitor groups was detected by CCK-8 assay. (D and E) Transwell migration and invasion assays detected the effects of miR-196b-5p on migratory and invasive abilities of A549 cells (I00x). All results were representative of three independent experiments. ${ }^{*} p<0.05, * * p<0.01$, $* * * p<0.001$.

that miR-196b-5p could specifically bind to the 3 '-UTR of WT-RSPO2 (Figure 3H). After overexpression or inhibition of miR-196b-5p expression in A549 cell line, the results of qRT-PCR (Figure 3I) and Western blot (Figure 3J) showed that compared with their respective control groups, mRNA and protein expression levels of RSPO2 were significantly decreased in A549 cells with overexpressed miR-196b-5p, and those were significantly increased in A549 cells with suppressed miR-196b-5p. These results suggested that miR-196b-5p could target and down-regulate the expression of RSPO2.

\section{Silencing RSPO2 Promotes Proliferation, Migration and Invasion of LUAD Cells}

To further explore the biological function of RSPO2 in LUAD, cell proliferative, migratory and invasive abilities were detected after silencing RSPO2 with siRNA. RSPO2 siRNA and NC siRNA were transfected into A549 cells, respectively. qRT-PCR and Western blot showed that transfection of RSPO2 siRNA effectively reduced the expression level of RSPO2 (Figure 4A and B). CCK-8 results indicated that silencing RSPO2 enhanced the proliferative capacity of A549 cells (Figure 4C). Transwell migration (Figure 4D) and invasion (Figure 4E) assays suggested that down-regulation of RSPO2 expression significantly promoted migration and invasion of A549 cells. These results demonstrated that silencing RSPO2 promoted proliferation, migration and invasion of LUAD cells.

\section{Overexpression of RSPO2 Can Reverse the Promotion of miR-196b-5p on Proliferation, Migration and Invasion of LUAD Cells to Some Extent}

NC-mimic +oe-NC, miR-196b-5p mimic +oe-NC or miR196b-5p mimic+oe-RSPO2 were transfected into A549 cells, and the interaction between miR-196b-5p and RSPO2 in LUAD cells was verified by detecting the mRNA and protein expression levels of RSPO2. Results of qRT-PCR (Figure 5A) and Western blot (Figure 5B) 

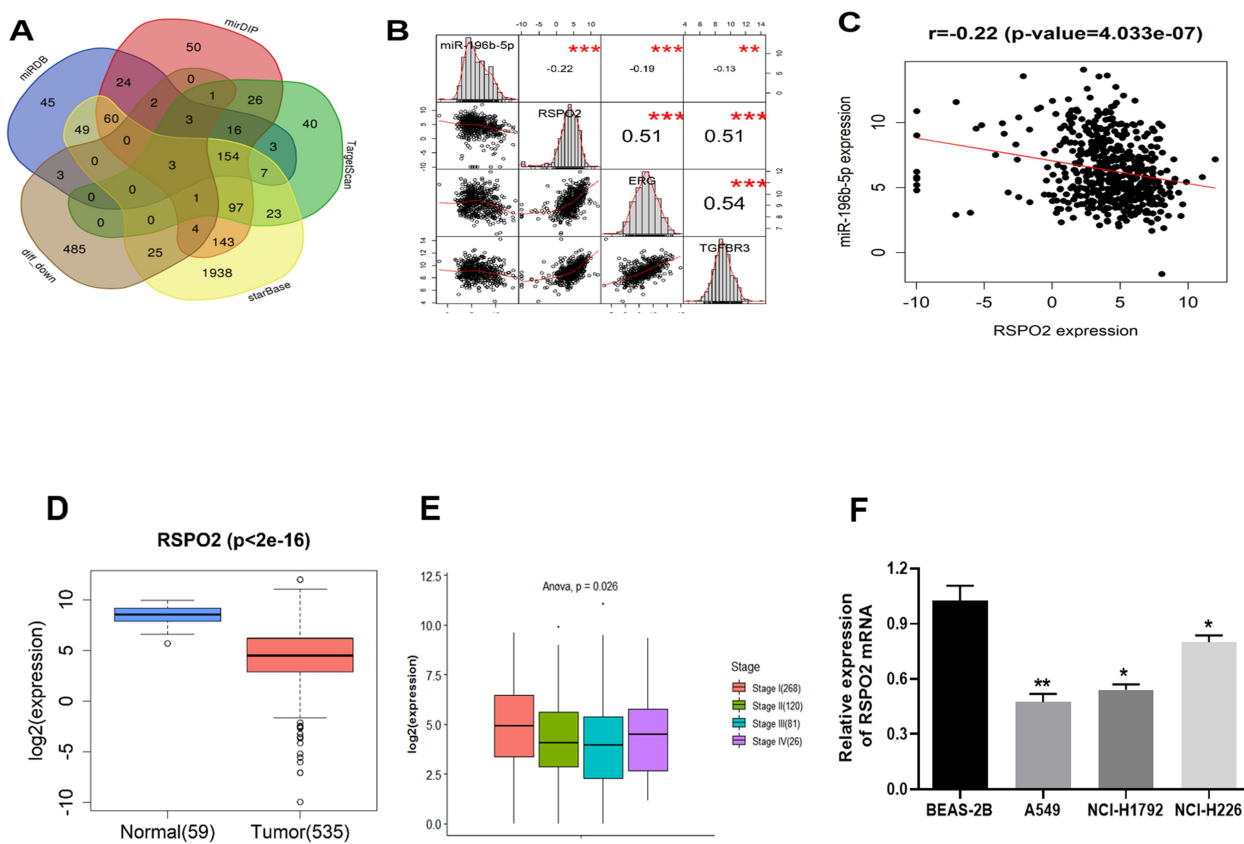

E

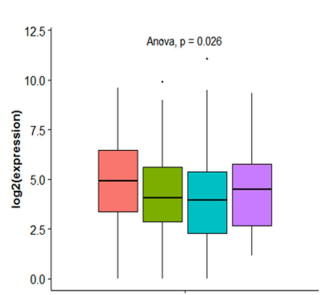

$\mathbf{F}$

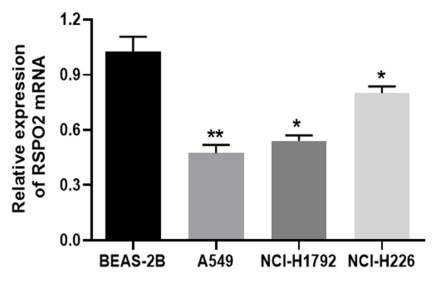

G
Wild position of RSPO2 3'UTR
5'..CCUUGCAUUGGCAAAACUACCUC..3'
hsa-miR-196b-5p
3' GgguUguUguccuUUGAUGGaU 5'

Mutant position of RSPO2 3'UTR 5'.CCUUGCAUUGGCAAAUGAUGGAC ·-3'
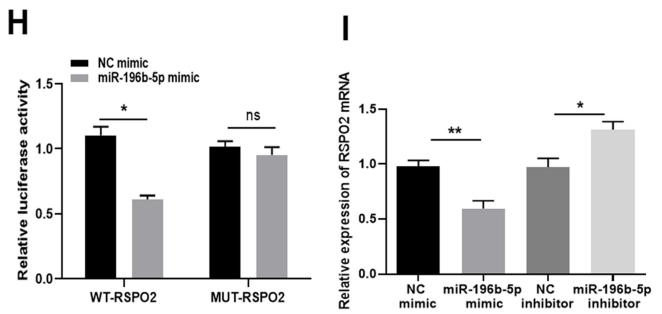

J

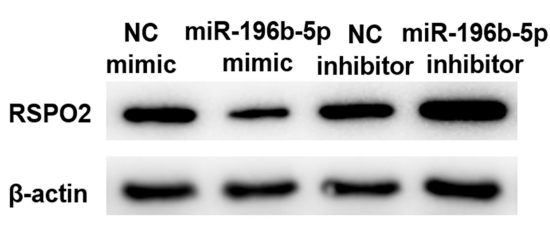

Figure 3 miR-196b-5p targets and down-regulates RSPO2. (A) The target genes of miR-196b-5p predicted by miRDB, mirDIP, TargetScan and starBase databases were intersected with down-regulated DEmRNAs. (B) Pearson correlation of miR-196b-5p and the candidate target genes TGFBR3, ERG and RSPO2. (C) Pearson correlation of miR-196b-5p and RSPO2. (D) The box plot of RSPO2 expression in normal tissue (green) and tumor tissue (red). (E) The correlation of RSPO2 and clinical stage. (F) The mRNA expression of RSPO2 in human normal bronchial epithelial cell line and three LUAD cell lines was detected by qRT-PCR. (G) The putative binding sites of RSPO2 3'UTR and miR-196b-5p. (H) The luciferase activity was detected by dual-luciferase reporter gene assay. (I and J) The mRNA and protein expression levels of RSPO2 were analyzed by qRT-PCR and Western blot in A549 cells transfected with miR-196b-5p mimic or inhibitor. All results were representative of three independent experiments. $* p<0.05, * * p<0.01$.

displayed that, compared with the control group, mRNA and protein expression levels of RSPO2 were significantly reduced in the miR-196b-5p mimic+oe-NC group, while those were reversed to increase significantly in miR-196b$5 \mathrm{p}$ mimic + oe-RSPO2 group. CCK-8 (Figure 5C) and transwell assays (Figure 5D and E) detected the proliferation, migration and invasion of the above transfected cells, and the results showed that overexpression of miR-196b$5 p$ dramatically enhanced proliferation, migration and invasion of LUAD cells compared with the control group. While overexpression of RSPO2 reversed such effects caused by miR-196b-5p overexpression. The above experimental results indicated that overexpression of RSPO2 reversed the promotion of miR-196b-5p on 
proliferation, migration and invasion of A549 cells to some extent.

\section{Discussion}

Lung cancer is a serious threat to human health. It is particularly important to find strategies for the diagnosis and treatment of lung cancer. Recent molecular targeted therapy for lung cancer has made great progress, and the research on miRNAs has achieved remarkable success. Kong et al discovered that miR-141 is significantly highly expressed in NSCLC, and promotes proliferation and invasion of lung cancer cells by targeting and down-regulating KLF9. ${ }^{20}$ Xiao pointed out that miR-7-5p is significantly down-regulated in NSCLC and inhibits tumor metastasis both in vivo and in vitro by targeting NOVA2, suggesting that miR-7-5p may be a novel biomarker and therapeutic target. $^{21}$ A study also has reported that miR-191-5p is overexpressed in lung cancer, and may block migration and proliferation of lung cancer cells through downregulating Wnt signaling via targeting SATB1. ${ }^{22}$ The above studies further indicate that miRNAs are significant for the development of lung cancer.

MiRNAs have been found to be aberrantly expressed in a variety of tumors, and they exert their role by targeting specific target genes. ${ }^{23}$ Among miRNAs, miR-196b-5p has been proved to be overexpressed in multiple tumors. Zhang et al put forward that miR-196b-5p is highly expressed in hepatocellular carcinoma tissue, and its expression level is related to the clinicopathological parameters such as blood vessel infiltration. ${ }^{24}$ Shao et al found that the up-regulation of miR-196b-5p in gastric cancer can promote proliferation and invasion of gastric cancer
A

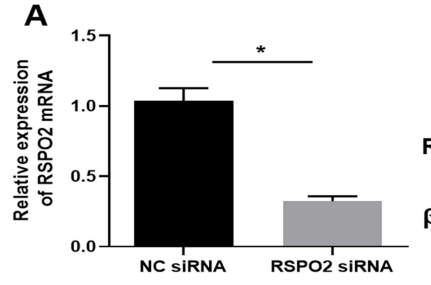

B

$\beta$-actin

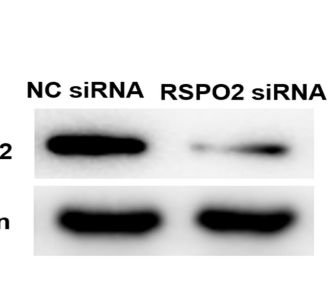

C

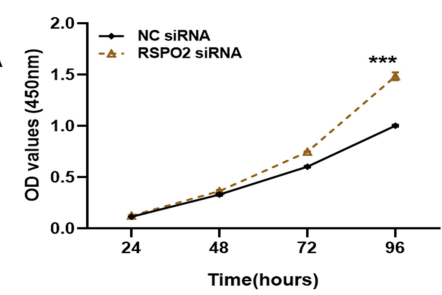

D

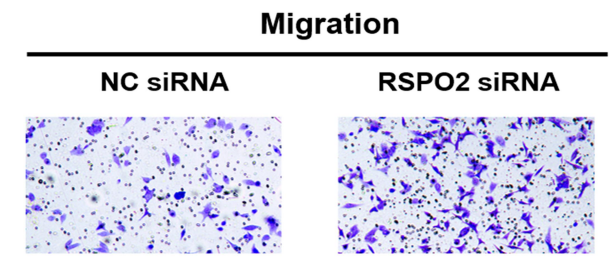

E

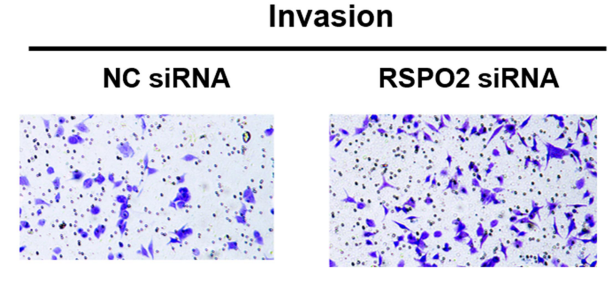

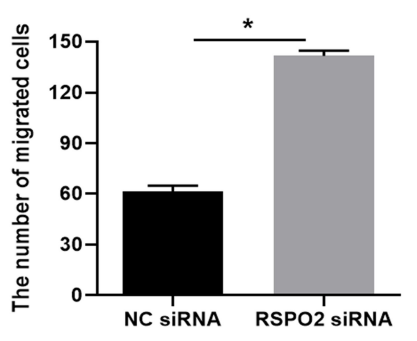

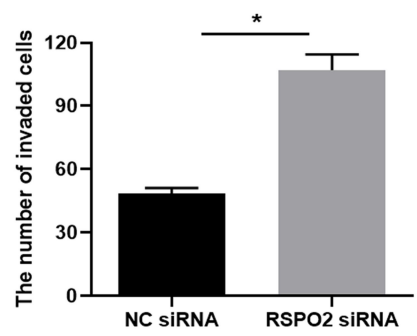

Figure 4 Silencing RSPO2 promotes proliferation, migration and invasion of LUAD cells. (A and B) The mRNA and protein expression levels of RSPO2 in A549 cells transfected with RSPO2 siRNA were detected by qRT-PCR and Western blot. (C) Cell viability was determined by CCK-8 after RSPO2 was silenced. (D and E) Transwell migration and invasion assays detected migratory and invasive capacities of $A 549$ cells $(100 \times)$. All results were representative of three independent experiments. * $p<0.05$, $* * * p<0.001$. 


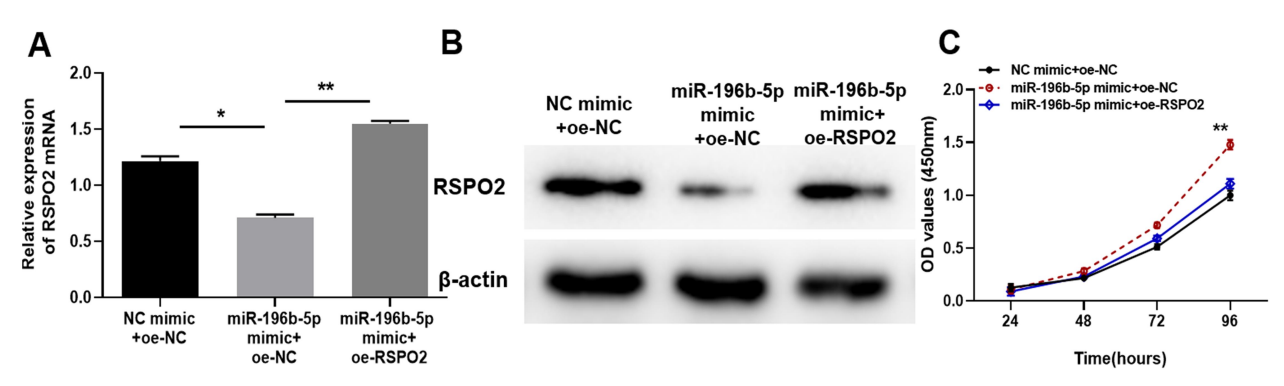

D

Migration

\begin{tabular}{ccc}
\hline $\begin{array}{c}\text { NC mimic } \\
\text { +oe-NC }\end{array}$ & $\begin{array}{c}\text { miR-196b-5p } \\
\text { mimic+oe-NC }\end{array}$ & $\begin{array}{c}\text { miR-196b-5p } \\
\text { mimictoe-RSPO2 }\end{array}$ \\
& & \\
& &
\end{tabular}

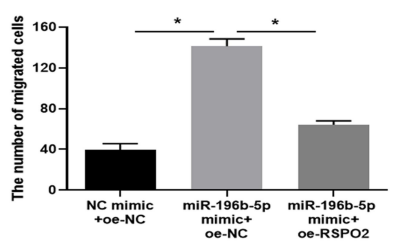

$\mathbf{E}$

\begin{tabular}{ccc}
\multicolumn{3}{c}{ Invasion } \\
\hline $\begin{array}{c}\text { NC mimic } \\
\text { +oe-NC }\end{array}$ & $\begin{array}{c}\text { miR-196b-5p } \\
\text { mimictoe-NC }\end{array}$ & $\begin{array}{c}\text { miR-196b-5p } \\
\text { mimic+oe-RSPO2 }\end{array}$ \\
& & \\
$-1+3$ & &
\end{tabular}

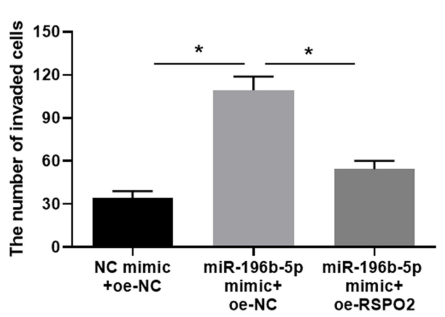

Figure 5 Overexpression of RSPO2 reverses the promotion of miR-196b-5p on proliferation, migration and invasion of LUAD cells. (A and B) qRT-PCR and Western blot were used to analyze RSPO2 mRNA and protein expression levels in NC mimic+oe-NC, miR-196b-5p mimic +oe-NC and miR-196b-5p mimic+oe-RSPO2 groups. (C) CCK8 detected the proliferative capacity of cells in each group. (D and E) Transwell migration and invasion assays detected the migratory and invasive capacities of A549 cells in each group (100×). All results were representative of three independent experiments. $* p<0.05, * * p<0.01$.

cells. ${ }^{25}$ Fantini et al reported that miR-196b is significantly up-regulated and promotes the development of colon cancer by targeting and down-regulating GATA6. ${ }^{26}$ In addition, miR-196b-5p is also significantly up-regulated in NSCLC ${ }^{27}$ laryngeal squamous cell carcinoma ${ }^{28}$ and pancreatic cancer. ${ }^{29}$ In this study, various experimental results illustrated that miR-196b-5p was overexpressed in LUAD tissue and cells, and promoted proliferation, migration and invasion of LUAD cells. The above studies strongly support our findings on the expression pattern and biological function of miR-196b-5p in LUAD.

Herein, after prediction and experimental verification, it was first found that RSPO2 was the direct target of miR-196b-
$5 p$. RSPO2 is an important member of the R-spondin family of secreted proteins, an important class of Wnt pathway activators discovered in recent years, ${ }^{18}$ while Wnt signaling pathway has been widely believed to be closely related to tumorigenesis and development. In recent years, many studies have pointed out that RSPO2 is overexpressed in thyroid cancer, ${ }^{30}$ gastric cancer, ${ }^{18}$ squamous cell carcinoma ${ }^{31}$ and other cancers, and up-regulation of RSPO2 will promote the progression of these cancers. However, some studies have also pointed out that upregulated RSPO2 has an anticancer effect in some cancers. Dong et al revealed that RSPO2 inhibits colorectal cancer (CRC) metastasis by counteracting the Wnt5a/Fzd7-driven noncanonical Wnt signaling pathway, and that down- 
regulation of RSPO2 expression promotes tumor metastasis and predicts a poor survival in CRC patients. ${ }^{32} \mathrm{Wu}$ et al demonstrated for the first time that there is a positive effect of RSPO2 high expression on LUAD patients and high expression of RSPO2 predicts the improvement of overall survival of LUAD patients, which may play an important protective role in lung cancer. ${ }^{13}$ The above studies provide a basis for our conclusion that RSPO2 as a tumor suppressor promoted proliferation, migration and invasion of LUAD cells upon its low expression.

After confirmed the targeted relationship between RSPO2 and miR-196b-5p, we conducted rescue experiments to further verify the interaction between miR196b-5p and RSPO2 in LUAD. It was concluded that the up-regulation of miR-196b-5p inhibited the expression of RSPO2, promoted proliferation, migration and invasion of LUAD cells, while overexpression of RSPO2 could reverse the promotion of miR-196b-5p on LUAD cells to some extent. miR-196b has also reported to promote migration and invasion of lung cancer cells by targeting and down-regulating GATA6, and the overexpression of GATA6 can also partially attenuate the carcinogenic effect of miR-196b up-regulation on LUAD cells. ${ }^{27}$

This study reported for the first time the effect of the miR-196b-5p/RSPO2 regulatory axis on the occurrence and development of LUAD. miR-196b-5p promotes proliferation and invasion of LUAD cells by directly targeting and downregulating RSPO2. This study indicates that miR-196b-5p is expected to be a diagnostic biomarker and a therapeutic target for LUAD. Nevertheless, the downstream molecular mechanism of the miR-196b-5p/RSPO2 regulatory axis in LUAD will be further explored in future experiments.

\section{Highlights}

1. miR-196b-5p was highly expressed in lung adenocarcinoma (LUAD);

2. miR-196b-5p promoted proliferation, migration and invasion of LUAD cells;

3. miR-196b-5p targeted and down-regulated RSPO2 in LUAD;

4. Silencing RSPO2 promoted proliferation, migration and invasion of LUAD cells;

5. Overexpression of RSPO2 could reverse the promoting effect of miR-196b-5p on proliferation, migration and invasion of LUAD cells.

\section{Disclosure}

The authors report no conflicts of interest for this work.

\section{References}

1. Liu W, Xie X, Wu J. Mechanism of lung adenocarcinoma spine metastasis induced by CXCL17. Cell Oncol (Dordr). 2020;43:311-320. doi:10.1007/s13402-019-00491-7

2. Torre LA, Bray F, Siegel RL, et al. Global cancer statistics, 2012. CA Cancer J Clin. 2015;65(2):87-108. doi:10.3322/caac.21262

3. Bray F, Ferlay J, Soerjomataram I, et al. Global cancer statistics 2018: GLOBOCAN estimates of incidence and mortality worldwide for 36 cancers in 185 countries. CA Cancer J Clin. 2018;68 (6):394-424. doi:10.3322/caac. 21492

4. Casali C, Rossi G, Marchioni A, et al. A single institution-based retrospective study of surgically treated bronchioloalveolar adenocarcinoma of the lung: clinicopathologic analysis, molecular features, and possible pitfalls in routine practice. $J$ Thorac Oncol. 2010;5:830-836. doi:10.1097/jto.0b013e3181d60ff5

5. Kim HC, Jung CY, Cho DG, et al. Clinical characteristics and prognostic factors of lung cancer in Korea: a pilot study of data from the Korean Nationwide Lung Cancer Registry. Tuberc Respir Dis (Seoul). 2019;82:118-125. doi:10.4046/trd.2017.0128

6. Valentino F, Borra G, Allione P, Rossi L. Emerging targets in advanced non-small-cell lung cancer. Future Oncol. 2018;14:61-72. doi:10.2217/fon-2018-0099

7. Zhang L, Huang P, Li Q, Wang D, Xu CX. miR-134-5p promotes stage I lung adenocarcinoma metastasis and chemoresistance by targeting DAB2. Mol Ther Nucleic Acids. 2019;18:627-637. doi:10.1016/j.omtn.2019.09.025

8. Zhuang L, Shou T, Li K, et al. MicroRNA-30e-5p promotes cell growth by targeting PTPN13and indicates poor survival and recurrence in lung adenocarcinoma. J Cell Mol Med. 2017;21:2852-2862. doi: $10.1111 / \mathrm{jcmm} .13198$

9. Dai B, Kong DL, Tian J, et al. microRNA-1205 promotes cell growth by targeting APC2 in lung adenocarcinoma. Eur Rev Med Pharmacol Sci. 2019;23:1125-1133. doi:10.26355/eurrev_201902_17003

10. Liu K, Zhang W, Tan J, Ma J, Zhao J. MiR-200b-3p functions as an oncogene by targeting ABCA1 in lung adenocarcinoma. Technol Cancer Res Treat. 2019;18:1533033819892590. doi:10.1177/ 1533033819892590

11. Xiao X, Yang D, Gong X, et al. miR-1290 promotes lung adenocarcinoma cell proliferation and invasion by targeting SOCS4. Oncotarget. 2018;9(15):11977-11988. doi:10.18632/oncotarget.24046

12. Fan G, Xu P, Tu P. MiR-1827 functions as a tumor suppressor in lung adenocarcinoma by targeting MYC and FAM83F. J Cell Biochem. 2020;121:1675-1689. doi:10.1002/jcb.29402

13. Wu L, Zhang W, Qian J, et al. R-spondin family members as novel biomarkers and prognostic factors in lung cancer. Oncol Lett. 2019;18:4008-4015. doi:10.3892/ol.2019.10778

14. Kazanskaya O, Ohkawara B, Heroult M, et al. The Wnt signaling regulator R-spondin 3 promotes angioblast and vascular development. Development (Cambridge, England). 2008;135 (22):3655-3664. doi:10.1242/dev.027284

15. Han T, Schatoff EM, Murphy C, et al. R-Spondin chromosome rearrangements drive Wnt-dependent tumour initiation and maintenance in the intestine. Nat Commun. 2017;8(1):15945. doi:10.1038/ncomms15945

16. Bell SM, Schreiner CM, Wert SE, et al. R-spondin 2 is required for normal laryngeal-tracheal, lung and limb morphogenesis. Development (Cambridge, England). 2008;135(6):1049-1058. doi:10.1242/dev.013359

17. Yin $\mathrm{X}$, Yi H, Wang L, et al. R-spondin 2 promotes proliferation and migration via the $\mathrm{Wnt} / \beta$-catenin pathway in human hepatocellular carcinoma. Oncol Lett. 2017;14(2):1757-1765. doi:10.3892/ ol.2017.6339

18. Zhang H, Han X, Wei B, et al. RSPO2 enhances cell invasion and migration via the $\mathrm{WNT} / \beta$-catenin pathway in human gastric cancer. J Cell Biochem. 2019;120(4):5813-5824. doi:10.1002/jcb.27867 
19. Wu C, Qiu S, Lu L, et al. RSPO2-LGR5 signaling has tumour-suppressive activity in colorectal cancer. Nat Commun. 2014;5:3149. doi:10.1038/ncomms4149

20. Kong YJ, Tan XX, Zhang Y, et al. MiR-141 promotes cell proliferation and invasion in non-small cell lung cancer by targeting KLF9. Eur Rev Med Pharmacol Sci. 2019;23:10370-10378. doi:10.26355/ eurrev_201912_19676

21. Xiao H. MiR-7-5p suppresses tumor metastasis of non-small cell lung cancer by targeting NOVA2. Cell Mol Biol Lett. 2019;24:60. doi:10.1186/s11658-019-0188-3

22. Zhou LY, Zhang FW, Tong J, Liu F. MiR-191-5p inhibits lung adenocarcinoma by repressing SATB1 to inhibit Wnt pathway. Mol Genet Genom Med. 2020;8:e1043. doi:10.1002/mgg3.1043

23. Zhong J, Ren X, Chen Z, et al. miR-21-5p promotes lung adenocarcinoma progression partially through targeting SET/TAF -I $\alpha$. Life Sci. 2019;231:116539. doi:10.1016/j.lfs.2019.06.014

24. Zhang L, Luo B, Dang YW, et al. Clinical significance of microRNA-$196 \mathrm{~b}-5 \mathrm{p}$ in hepatocellular carcinoma and its potential molecular mechanism. J Cancer. 2019;10(22):5355-5370. doi:10.7150/jca.29293

25. Shao L, Chen Z, Peng D, et al. Methylation of the HOXA10 promoter directs miR-196b-5p-dependent cell proliferation and invasion of gastric cancer cells. Mol Cancer Res. 2018;16:696-706. doi:10.1158/1541-7786.Mcr-17-0655

26. Fantini S, Salsi V, Reggiani L, Maiorana A, Zappavigna V. The miR-196b miRNA inhibits the GATA6 intestinal transcription factor and is upregulated in colon cancer patients. Oncotarget. 2017;8:4747-4759. doi:10.18632/oncotarget.13580
27. Li H, Feng C, Shi S. miR-196b promotes lung cancer cell migration and invasion through the targeting of GATA6. Oncol Lett. 2018;16:247-252. doi:10.3892/ol.2018.8671

28. Zhao X, Zhang W, Ji W. miR-196b is a prognostic factor of human laryngeal squamous cell carcinoma and promotes tumor progression by targeting SOCS2. Biochem Biophys Res Commun. 2018;501:584-592. doi:10.1016/j.bbrc.2018.05.052

29. Kanno S, Nosho K, Ishigami K, et al. MicroRNA-196b is an independent prognostic biomarker in patients with pancreatic cancer. Carcinogenesis. 2017;38(4):425-431. doi:10.1093/carcin/bgx013

30. Kang YE, Kim JM, Kim KS, et al. Upregulation of RSPO2-GPR48/LGR4 signaling in papillary thyroid carcinoma contributes to tumor progression. Oncotarget. 2017;8(70):114980-114994. doi:10.18632/oncotarget.22692

31. Zhang L, Song Y, Ling Z, et al. R-spondin 2-LGR4 system regulates growth, migration and invasion, epithelial-mesenchymal transition and stem-like properties of tongue squamous cell carcinoma via Wnt/ $\beta$-catenin signaling. EBioMedicine. 2019;44:275-288. doi:10.1016/j.ebiom.2019.03.076

32. Dong X, Liao W, Zhang L, et al. RSPO2 suppresses colorectal cancer metastasis by counteracting the Wnt5a/Fzd7-driven noncanonical Wnt pathway. Cancer Lett. 2017;402:153-165. doi:10.1016/j. canlet.2017.05.024

\section{Publish your work in this journal}

Cancer Management and Research is an international, peer-reviewed open access journal focusing on cancer research and the optimal use of preventative and integrated treatment interventions to achieve improved outcomes, enhanced survival and quality of life for the cancer patient.
The manuscript management system is completely online and includes a very quick and fair peer-review system, which is all easy to use. Visit http://www.dovepress.com/testimonials.php to read real quotes from published authors. 